\title{
Perfil epidemiológico das fraturas de falange de pacientes internados em um Hospital do Norte de Minas Gerais
}

\author{
Epidemiological profile of phalanx fractures in patients admitted to a Hospital in the North \\ of Minas Gerais
}
Perfil epidemiológico de las fracturas de falange en pacientes ingresados en un Hospital del Norte de Minas Gerais

Thyago Bacelar Vieira ${ }^{1 *}$, Renato Medeiros Aguiar ${ }^{1}$, Samuel de Paiva Oliveira ${ }^{2}$, Alexander Rocha Siqueira², Gabriela Barbosa Silva², Ana Júlia Soares Oliveira², Rodrigo Braulio Franca Madureira².

\section{RESUMO}

Objetivo: Analisar as características epidemiológicas associadas às fraturas de falanges dos dedos da mão. Métodos: Pesquisa de caráter epidemiológico, com delineamento documental, retrospectivo, descritivo e analítico. Amostra inicial referente aos atendimentos, entre janeiro de 2018 e dezembro de 2020, de 331 pacientes com fraturas de membros superiores cirúrgicas, realizando a evolução das suas variáveis a partir de um formulário sociodemográfico, com posterior análise dos dados estatísticos pelo programa IBM SPSS Statistics. Estudo foi aprovado por Comitê de Ética em Pesquisa. Resultados: Selecionou-se 42 prontuários de pacientes com fratura de falange, em meio aos 331 casos atendidos, sendo 20 proximal, 10 média e 12 distal. A amostra apresenta maioria do sexo masculino (100\%, $90 \%$ e $83,3 \%$ respectivamente), na faixa etária de 18 a 60 anos $(75 \%, 90 \%$ e 66,6\%). Evidencia como principal mecanismo de trauma o acidente automobilístico $(26,2 \%)$, pequena incidência de refraturas $(4,8 \%)$, tempo de internação majoritariamente inferior a uma semana $(60 \%)$ e poucas complicações pós-cirúrgicas (9,6\%). Empregou-se a osteossíntese como abordagem cirúrgica, apresentando como único tipo de implante o Fio de Kirchner. Conclusão: Concluise que o estudo revelou um perfil epidemiológico relativamente semelhante ao descrito na literatura disponível, diferenciando-se apenas na maior prevalência das fraturas de falange proximal.

Palavras-chave: Falange dos dedos da mão, Fraturas ósseas, Epidemiologia descritiva, Fixação interna de fraturas.

\begin{abstract}
Objective: To analyze the epidemiological characteristics associated with phalangeal fractures of the fingers. Methods: Epidemiological research, with documentary, retrospective, descriptive and analytical design. Initial sample referring to care, between January 2018 and December 2020, of 331 patients with surgical upper limb fractures, performing the evolution of their variables from a sociodemographic form, with subsequent analysis of statistical data by the IBM SPSS Statistics program. Study was approved by the Research Ethics Committee. Results: Forty-two medical records of patients with phalanx fractures were selected, among the 331 cases treated, being 20 proximal, 10 medium and 12 distal. The sample is mostly male $(100 \%, 90 \%$ and $83.3 \%$ respectively), aged between 18 and 60 years (75\%, 90\% and $66.6 \%)$. It shows as the main trauma mechanism the car accident $(26.2 \%)$, low incidence of refractures (4.8\%), hospital stay mostly less than one week $(60 \%)$ and few post-surgical complications $(9.6 \%)$. Osteosynthesis was used as a surgical approach, with the
\end{abstract}

${ }^{1}$ Hospital Aroldo Tourinho, Montes Claros - MG. *E-mail: thyagob.vieira@hotmail.com

2 Universidade Estadual de Montes Claros (UNIMONTES), Montes Claros - MG. 
Kirchner wire as the only type of implant. Conclusion: It is concluded that the study revealed an epidemiological profile relatively similar to that described in the available literature, differing only in the higher prevalence of fractures of the proximal phalanx.

Keywords: Finger phalanges, Fractures bone, Epidemiology descriptive, Fracture fixation internal.

\section{RESUMEN}

Objetivo: Analizar las características epidemiológicas asociadas a las fracturas de falange de los dedos. Métodos: Investigación epidemiológica, con diseño documental, retrospectivo, descriptivo y analítico. Muestra inicial referente a la atención, entre enero de 2018 y diciembre de 2020, de 331 pacientes con fracturas quirúrgicas de miembro superior, realizando la evolución de sus variables desde una forma sociodemográfica, con posterior análisis de datos estadísticos por el programa IBM SPSS Statistics. El estudio fue aprobado por el Comité de Ética en Investigación. Resultados: Se seleccionaron 42 historias clínicas de pacientes con fracturas de falange, entre los 331 casos tratados, siendo 20 proximales, 10 medianos y 12 distales. La muestra es mayoritariamente masculina (100\%, $90 \%$ y $83,3 \%$ respectivamente), con edades comprendidas entre 18 y 60 años $(75 \%, 90 \%$ y 66,6\%). Muestra como principal mecanismo traumático el accidente automovilístico $(26,2 \%)$, baja incidencia de refracturas $(4,8 \%)$, estancia hospitalaria mayoritariamente menor a una semana $(60 \%)$ y pocas complicaciones posquirúrgicas $(9,6 \%)$. La osteosíntesis se utilizó como abordaje quirúrgico, siendo el alambre de Kirchner el único tipo de implante. Conclusión: Se concluye que el estudio reveló un perfil epidemiológico relativamente similar al descrito en la literatura disponible, difiriendo solo en la mayor prevalencia de fracturas de la falange proximal.

Palabras clave: Falanges de los dedos de la mano, Fracturas ósseas, Epidemiología descriptiva, Fijación interna de fracturas.

\section{INTRODUÇÃO}

No pronto-atendimento, fraturas da mão são queixas comuns, representando cerca de $10 \%$ de todas as fraturas avaliadas. Essas lesões têm o potencial de gerar complicações e consequências significativas, principalmente, para a população jovem e economicamente ativa em que predominam. Então, é notória sua relevância na saúde pública, sendo fundamental a compreensão dos fatores epidemiológicos associados, especialmente, nas fraturas de falanges dos dedos da mão (KEE, et al., 2021).

Apesar da relevância médica considerável, nota-se escassas publicações científicas voltadas para a epidemiologia das fraturas de falange dos dedos da mão, particularmente, no Brasil. Segundo Liu S, et al. (2021), as fraturas de mão e de punho representam cerca de $28,6 \%$ de todas as fraturas. Conforme Karl JW, et al. (2015), em estudo conduzido nos Estados Unidos em 2009, as fraturas de falanges afetam 12,5 por 10.000 pessoas-ano. Taha $\mathrm{R}$, et al. (2021) descreveram que os metacarpos foram mais frequentemente fraturados do que as falanges como um grupo combinado, sendo que a maioria de todas as fraturas da mão analisadas foram extra-articulares. Fraturas intra-articulares e pacientes com fraturas múltiplas apresentaram pouco frequência, mas foram mais provavelmente tratadas de forma cirúrgica.

O perfil do paciente com maior risco de fraturas de falange deve considerar, principalmente, a faixa etária e o gênero, uma vez que homens jovens e as mulheres idosas são mais suscetíveis a essa lesão. $O$ aumento dos fatores de risco comportamentais, que incluem a participação em esportes e as atividades laborais, justificam que o sexo masculino permaneça a um nível relativamente maior de risco de fraturas de falange até a idade de aposentadoria, aos $60-65$ anos de idade. As mulheres apresentam maior risco de fratura de falange dos dedos da mão após os 65 anos de idade, o que pode estar associado a uma elevada expectativa de vida e a um aumento exponencial na incidência de quedas e de osteoporose (KREMER L, et al., 2020; MEALS C e MEALS R, 2013; RIBAK S, et al., 2018).

Em relação aos mecanismos de lesão mais frequentes, considera-se o golpe direto e queda acidental, variando conforme as diferentes faixas etárias. Karl JW, et al. (2015) evidenciaram que as quedas são 
responsáveis pela maioria das fraturas de falange em pacientes acima de 70 anos. Kremer L, et al. (2020) demonstraram que pacientes adultos sofreram principalmente lesões por colisão/golpe direto, enquanto quedas acidentais predominaram em pacientes idosos.

Portanto, entende-se que em sociedades altamente especializadas muitas atividades diárias exigem habilidades manuais, ou seja, injúrias nas mãos causarão grandes dificuldades para indivíduos de todas as idades. Do ponto de vista da saúde pública, as fraturas nas mãos, especialmente de falange, requerem atenção médica urgente, uma vez que acarretam grandes custos para o sistema de saúde e para o sistema social (RIBAK S, et al., 2018).

Entretanto, as informações epidemiológicas atuais sobre lesões nas mãos que chegam à assistência médica intensiva na população em geral são insuficientes. Assim, o objetivo desse estudo foi analisar as características epidemiológicas associadas às fraturas de falanges dos dedos da mão atendidas cirurgicamente em um Hospital no Norte de Minas Gerais.

\section{MÉTODOS}

Trata-se de um recorte de um estudo de caráter epidemiológico, com delineamento documental, retrospectivo, descritivo e analítico, denominado: "Características epidemiológicas das fraturas de membros superiores cirúrgicas em um hospital do Norte de Minas Gerais". Na área de urgência e emergência, o hospital em questão classifica-se como trauma nível dois, apresentando o serviço de pronto-atendimento por 24 horas, sendo a ortopedia como uma das especialidades oferecidas. Além disso, destaca-se que a prestação de serviços ocorre integralmente pelo Sistema único de Saúde (SUS), associando-se às atividades de ensino, de pesquisa e de extensão desenvolvidas no local.

Em relação à coleta dos dados, realizou-se a partir das informações provenientes do sistema de informação interno da instituição, sendo conduzida de forma presencial, em local e horário previamente agendados pela equipe, que foi anteriormente treinada e calibrada para realizar a extração dos dados a partir da análise dos prontuários e protocolos de coleta pré-estabelecidos. A amostra populacional foi composta por 331 pacientes que foram atendidos em um Hospital no Norte de Minas Gerais entre janeiro de 2018 e dezembro de 2020.

Neste estudo, incluiu-se pacientes adultos do sexo feminino e do sexo masculino, acima de 18 anos de idade, atendidos pelo serviço cirúrgico com fraturas de membros superiores entre janeiro de 2018 e dezembro de 2020 em um Hospital do Norte de Minas Gerais. Excluiu-se pacientes abaixo de 18 anos de idade, além daqueles cujos prontuários apresentavam dados incompletos. Utilizou-se um formulário sociodemográfico para a evolução (retrospectiva) das variáveis. Os dados foram analisados através da estatística descritiva (frequência simples e relativa), utilizando o programa Statical Package for Social Science (SPSS), versão 20.0 .

Evidencia-se que esta pesquisa não incluiu um Termo de Consentimento Livre e Esclarecido, pois os dados foram de obtidos de maneira retrospectiva, secundária, de pacientes que foram atendidos ou que vieram a óbito, respeitando as diretrizes da resolução CNS $N^{\circ} 466$ de 2012. O projeto de pesquisa foi aprovado pelo Comitê de Ética em Pesquisa da Universidade Estadual de Montes Claros (UNIMONTES) por meio do parecer consubstanciado 4.838.958.

\section{RESULTADOS}

Os dados foram coletados da data de janeiro de 2018 a dezembro de 2020, e foram selecionados 42 pacientes com fratura de falange para esse estudo. Todos os prontuários dos pacientes foram analisados pelo sistema informatizado do hospital. Para melhor discussão, as fraturas foram divididas em: proximal, com 20 (47,6\%); média, com 10 (23,8\%); distal, com 12 (28,6\%). Dentre os pacientes que tiveram fratura proximal, $15(75 \%)$ tinham entre 18 e 60 anos de idade, enquanto que $5(25 \%)$ eram maiores que 60 anos. Sobre a fratura média, $8(90 \%)$ tinham menos que 61 anos, e apenas $2(20 \%)$ tinham idade superior a 60 anos. Em relação à fratura de falange distal, $8(66,6 \%)$ tinham menos de 61 anos e $04(23,4 \%)$ tinham mais de 60 anos. 
Em relação ao sexo, todos os subtipos de fratura de falange (proximal, média e distal) tiveram um maior predomínio do sexo masculino, representado por 100\%, 90\% e 83,3\%, respectivamente (Tabela 1).

Tabela 1 - Relação entre o tipo de fratura de falange, o sexo e a idade.

\begin{tabular}{cccc}
\hline \multirow{2}{*}{ Variáveis } & \multicolumn{3}{c}{ Fratura de falange } \\
\cline { 2 - 4 } & Proximal & Média & Distal \\
\hline Idade & & & 5 \\
\hline $18-40$ & 8 & 7 & 3 \\
$41-60$ & 7 & 1 & 4 \\
$61-80$ & 5 & 2 & 0 \\
$>81$ & 0 & 0 & 10 \\
\hline Sexo & & & 2 \\
\hline Masculino & 20 & 9 & \\
\hline Feminino & 0 & 1 &
\end{tabular}

Fonte: Vieira TB, et al., 2021.

Em relação ao mecanismo de trauma, os mais frequentes foram o acidente automobilístico, representado por $11(26,2 \%)$, seguido por acidente de trabalho (19\%). Outros mecanismos de trauma que estavam presentes fora: acidente com arma branca, acidente doméstico, acidente com arma de fogo, atropelamento, esmagamento, queda da própria altura e trauma contuso. Ressalta-se que, dentre os prontuários analisados, $11(26,2 \%)$ pacientes não tinham o mecanismo de trauma informado (Tabela 2).

Tabela 2 - Relação entre o tipo de fratura de falange e o mecanismo de trauma apresentado.

\begin{tabular}{lccc}
\hline \multirow{2}{*}{ Variáveis } & \multicolumn{3}{c}{ Fratura de falange } \\
\cline { 2 - 4 } & Proximal & Média & Distal \\
\hline Mecanismo de Trauma & 3 & 4 & 4 \\
\hline Acidente automobilístico & 1 & 0 & 0 \\
Acidente com arma branca & 4 & 1 & 3 \\
Acidente de trabalho & 0 & 1 & 0 \\
Acidente doméstico & 1 & 0 & 0 \\
Acidente com arma de fogo & 1 & 0 & 0 \\
Atropelamento & 2 & 1 & 1 \\
Esmagamento & 0 & 0 & 1 \\
Queda da própria altura & 2 & 0 & 1 \\
Trauma contuso & 6 & 3 & 2 \\
Não informado & & & \\
\hline
\end{tabular}

Fonte: Vieira TB, et al., 2021.

Outro ponto observado com a coleta de dados foi a relação entre o tipo de fratura com o tipo de cirurgia e o implante utilizado. O tipo de cirurgia mais realizado nos três casos (proximal, média e distal) foi a osteossíntese, representada por $85 \%, 100 \%$ e $83,3 \%$ dos casos. Em relação ao implante usado, o Fio de Kirschner foi o único implante descrito nos prontuários dos pacientes. Porém, 5 (12\%) prontuários não continham a informação sobre o implante utilizado (Tabela 3). 
Tabela 3 - Relação entre o tipo de fratura de falange, o tipo de cirurgia realizado e o implante utilizado.

\begin{tabular}{cccc}
\hline \multirow{2}{*}{ Variáveis } & \multicolumn{3}{c}{ Fratura de falange } \\
\cline { 2 - 4 } & Proximal & Média & Distal \\
\hline Tipo de Cirurgia & 3 & 0 & 2 \\
\hline Amputação & 17 & 10 & 10 \\
Osteossíntese & & & 11 \\
\hline Implante & 16 & 10 & 1 \\
\hline Fio de Kirschner & 4 & 0 &
\end{tabular}

Fonte: Vieira TB, et al., 2021.

Sobre o tempo de internação, 29 (69\%) pacientes ficaram menos de uma semana internados, 7 (16,6\%) ficaram uma semana, $6(14,3 \%)$ ficaram 2 semanas e $1(2,3 \%)$ ficou 3 semanas. Nenhum paciente ficou internado mais de 3 semanas.

Em relação à presença de refratura ou não, verificou-se que 40 (95,2\%) pacientes não apresentaram refratura. Quanto às complicações pós-cirúrgicas, 38 (90,4\%) pacientes não tiveram nenhuma complicação pós-cirúrgica, e teve uma incidência mínima de amputação; infecção da fratura exposta; isquemia e amputação; e lesão do tendão extensor; cada uma com 1 caso (2,4\% cada) (Tabela 4).

Tabela 4 - Relação entre o tipo de fratura de falange, o tempo de internação hospitalar, a presença de refratura e as complicações pós-cirúrgicas.

\begin{tabular}{lccc}
\hline \multirow{2}{*}{ Variáveis } & \multicolumn{3}{l}{ Fratura de falange } \\
\cline { 2 - 4 } & Proximal & Média & Distal \\
\hline Tempo de internação & 13 & 5 & 11 \\
\hline 1 semana & 4 & 2 & 1 \\
1 semana & 3 & 2 & 1 \\
2 semanas & 0 & 1 & 0 \\
3 semanas & 0 & 0 & 0 \\
1 mês & 0 & 0 & 0 \\
$>1$ mês & & & \\
\hline Refratura & 1 & 1 & 12 \\
\hline Sim & 19 & 9 & 11 \\
Não & & & 0 \\
Complicações & 18 & 9 & 0 \\
\hline Sem complicações & 0 & 0 & 0 \\
Amputação & 0 & 1 & 0 \\
Infecção da fratura exposta & 1 & 0 & \\
Isquemia e amputação & 1 & 0 & \\
Lesão de tendão extensor & & &
\end{tabular}

Fonte: Vieira TB, et al., 2021.

\section{DISCUSSÃO}

Dentre a complexidade do corpo humano, uma estrutura chama atenção pelas suas inúmeras funcionalidades, como a capacidade de captar e transmitir sensações e a execução de movimentos complexos e delicados. Essas funções permitem ao indivíduo desempenhar as mais diversas atividades 
essenciais à sua sobrevivência como a comunicação; realização de atividades laborais, identificação de objetos no espaço e até mesmo defesa pessoal. A estrutura em questão é a mão. Composta por 27 ossos e inúmeros músculos, ligamentos, tendões, vasos e nervos, as mãos, embora tão importantes para as atividades do dia a dia, são estruturas relativamente expostas e vulneráveis a lesões, lesões essas que constantemente ameaçam a capacidade funcional do indivíduo (KÖCHLI S, et al., 2020).

Nesse contexto, a literatura atual traz que as fraturas de falange ocupam o terceiro lugar na incidência de fraturas do membro superior, com picos na segunda década de vida no sexo feminino e terceira década no sexo masculino, sendo que os homens apresentam duas vezes mais risco de fratura falangiana do que as mulheres (WOLFE SW, et al., 2016). Tal prevalência apresenta certa semelhança ao que foi encontrado nesse estudo, isto é, há uma prevalência dos homens sobre às mulheres, porém na proporção aproximada de 11:1, com pico de incidência entre os 20-40 anos, com manutenção relativa deste pico até os 60 anos. Uma hipótese que ajuda a explicar essa ampla prevalência se dá pelo fato de que as vítimas dessas fraturas são indivíduos submetidos a mais riscos ocupacionais, uma vez que boa parte dos mecanismos de traumas dessas fraturas apresenta correlação direita com acidentes ocupacionais (BLAZAR PE e STEINBERG DR, 2016; SZEKERES M, et al., 2016).

Notoriamente, ocorre um atraso para realização do diagnóstico dessas fraturas. Como mencionado, os quirodáctilos são regiões bastante expostas e, consequentemente, susceptíveis a traumas. Como traumas de baixa cinética são comuns nessas estruturas, boa parte dos doentes não se atenta a possibilidade de fratura óssea, principalmente quanto estas são exarticulares, pois não costuma comprometer de imediato a funcionalidade dos dedos. Segundo Wolfe SW, et al. (2016), os pacientes tendem a recorrer ao serviço médico duas a quatro semanas após o evento traumático, principalmente pela persistência da sintomatologia como dor intensa e edema. Quando se trata das fraturas articulares, a situação é ainda mais complexa, pois estes dedos tendem a perda de funcionalidade por rigidez e artrose articular, sendo que para cada atraso no diagnóstico, há mais 100 casos de quirodáctilos com rigidez articular permanente decorrente de atrasos diagnósticos (HAWKEN JB e GILADI AM, 2021).

Outro aspecto considerável é a influência dos fatores intrínsecos ao indivíduo na ocorrência ou no sucessolfalha terapêutica dessas fraturas. A questão aqui destacada é a menor prevalência dessas fraturas em pacientes com idade maior que 65 anos, descrita tanto nos bancos de dados quanto neste estudo. $O$ fator senilidade quase não exerceu impacto na mecânica traumática ou na terapêutica desses indivíduos, diferentemente de lesões como as fraturas de Colles e as fraturas pertrocantéricas, as quais as comorbidades são um importante fator na ocorrência do trauma e no sucessolfalha do tratamento escolhido (ASPRAY TJ, et al., 2019).

Ao se realizar o traçado epidemiológico de fraturas em geral, a classificação das lesões ósseas costuma ser essencial para que se possa compreender a prevalência dos padrões fraturários naquela amostra e correlaciona-los com outros fatores como o mecanismo de trauma e as opções de abordagem terapêutica. Em geral, a classificação AO costuma servir muito bem a este propósito, entretanto, por apresentar uma baixa concordância interobservadores e intraobservadores nas fraturas de mão, esta é pouco adotada tanto nos estudos epidemiológicos quanto na pratica clínica (ROCKWOOD CA, et al., 2019). Dessa forma, as fraturas de falange desse estudo não foram classificadas com o sistema $A O$.

Considerando a epidemiologia das lesões falangianas distais, a literatura atual sugere que as fraturas de falange distal (F3) são responsáveis por mais da metade das fraturas de todos os ossos da mão (BLAZAR PE e STEINBERG DR, 2016). Um dos principais motivos para essa prevalência reside no fato de que as falanges distais são o ponto terminal de contato com o ambiente externo, sendo expostas a estresse mecânico praticamente a cada uso da mão pelo paciente (ROCKWOOD CA, et al., 2019). Ademais, esses ossos são as estruturas que mais se projetam "além do corpo", tornando-as extremamente expostas (WOLFE SW, et al., 2016).

A prova disso é que a falange distal do $3^{\circ}$ quirodáctilo é, epidemiologicamente a mais fraturada, pois é a mais exposta dentre todos os dedos das mãos. Entretanto tal dado não foi comprovado na análise amostral 
deste estudo. A segunda falange distal mais lesada é a do $1^{\circ}$ quirodáctilo, isso por que o polegar é essencial para a realização do movimento de pinça, movimento esse executada com alta frequência pelas mãos, aumentando a chance de traumas nessa região. Todavia, na contramão da literatura atual sobre o tema, neste estudo, as fraturas de falange distal apresentaram uma incidência ligeiramente menor que às fraturas de falange proximal. Um dos motivos que ajuda a explicar essa inversão epidemiológica é o fato de que este estudo se limitou às fraturas falangianas cirúrgicas. Dessa forma como as indicações de osteossíntese para as fraturas de F1 são mais amplas que as de F3, a tendencia é encontrar-se uma maior prevalência das fraturas de falange proximal nestas circunstâncias.

Ser o ponto terminal de contato com o meio ambiente torna as falanges distais susceptíveis a certos tipos de mecanismos traumáticos, como os esmagamentos. Enquanto nas crianças, a preensão do dedo no fechamento de portas é a principal etiologia traumática, nos adultos, o trauma ocupacional lidera a prevalência, principalmente nos homens (GERHARDT S e SILVA JB, 2014). Segundo Blazar PE e Steinberg DR (2016), às maquinas de rolo costumam ser a principal causa de fratura ocupacional de falange distal.

O manuseio de materiais pesados e de equipamentos de perfuração ocuparam a segunda e terceira posição de prevalência respectivamente. Quanto às fraturas complexas expostas, as atividades com equipamentos cortantes como serras ganham destaque. Na amostra desse estudo, os acidentes com maquita são apontados como a principal causa de fratura de cunho ocupacional, gerando em sua grande maioria, fraturas expostas. Entretanto, em distinção ao que é descrito na literatura, nesta amostra, os acidentes automobilísticos são o principal mecanismo traumático geral das fraturas de F3. As fraturas associadas a traumas esportivos, cuja incidência descrita é relativamente expressiva na literatura, não tiveram destaque nesse estudo, não sendo abordadas aqui (WOLFE SW, et al., 2016; PATEL L, 2014).

Nesse contexto de traumatismos de alta cinética, as lesões associadas às fraturas de F3 ganham destaque, principalmente as lesões nas regiões digitais e do aparelho ungueal. Sendo utilizadas como instrumento de defesa pessoal no passado, as unhas têm funções que vão muito além da proteção dos dedos ou de servir como complemento estético para as mulheres. Essa estrutura potencializa o tato epicrítico dos dedos, aumenta a capacidade discriminatória entre dois pontos e aperfeiçoa o fino movimento de pinça da mão. Dessa forma, as lesões do leito ungueal merecem tanta atenção quanto as lesões ósseas de F3 (SILVA JB e GERHARDT S, 2014).

$\mathrm{Na}$ abordagem terapêutica de fraturas de falange distal com lesão do aparelho ungueal, o manejo das lesões do complexo ungueal otimiza o resultado funcional da fratura de F3, sendo que lesões mal abordadas desse aparelho tendem a resultar em dor crônica e deformidades locais. Algumas opções de tratamento são a trepanação dos hematomas subungueais para alívio da dor local; a sutura solúvel dos leitos lacerados, o uso de adesivos de octil-2-cianoacrilato para cobertura do leito e, em casos mais graves, a substituição da lâmina ungueal (HAWKEN JB e GILADI AM, 2021). No serviço base deste estudo, não foi descrito a utilização de nenhuma das opções descritas acima em caso de lesões simples. Nos casos de laceração do local, sempre que possível, foi realizado a tentativa de manutenção do posicionamento da unha como forma de proteção óssea de $F 3$, até que outra unha cresça no local.

Quanto à abordagem das fraturas de $\mathrm{F} 3 \mathrm{em}$ si, neste estudo, a grande maioria não necessitou de osteossíntese para a consolidação óssea, mesmo naqueles casos de fratura exposta ou com cominuição distal. Para a definição da necessidade de osteossíntese, em geral, são considerados fatores como o traço da fratura, o desvio, acometimento articular e grau de cominuição (ROCKWOOD CA, et al., 2019).

No serviço base deste estudo, o acometimento articular foi o principal motivo para fixação das fraturas de F3, sendo o fio de Kirschner o implante de escolha para essas fraturas, garantindo a estabilidade necessária e com baixos percentuais de complicações. A principal complicação dessas fraturas de falange distal foi a infecção, associada ou não a necrose de partes moles, ainda sim, com baixa prevalência. A amputação parcial ou total da falange distal foi a opção terapêutica em $40 \%$ dos casos de fratura de $\mathrm{F} 3$, isto é, nos casos com grave comprometimento de partes moles e do suporte neurovascular, bem como nas perdas de substância 
óssea local. $O$ fechamento dessas amputações em geral foi feito por rafia primária, com baixos índices de complicações, semelhante ao que é descrito nas bases de dados sobre o tema (HAWKEN JB e GILADI AM, 2021).

Em se tratando das fraturas de falange média (F2), a incidência dessa lesão no contexto das fraturas falangianas cai bastante, principalmente se comparada a incidência das fraturas de F3. A distribuição quanto ao sexo e idade é semelhante ao das fraturas de falange distal. Quanto ao mecanismo de trauma, os bancos de dados também apontam para os acidentes ocupacionais como causa principal, entretanto, neste estudo, os acidentes automobilísticos também apresentam prevalência ligeiramente maior aos traumas ocupacionais (BLAZAR PE e STEINBERG DR, 2016). Até a prevalência do dedo atingido se assemelha ao das fraturas de falange distal, sendo que as falanges médias do $2^{\circ}$ e $3^{\circ}$ quirodáctilos foram as mais atingidas, neste estudo.

$\mathrm{O}$ que difere das fraturas de F3 é, principalmente, a abordagem terapêutica. Aqui, o tratamento com osteossíntese é mais comum. Segundo Rockwood CA, et al. (2019), as forças deformantes das fraturas de F2 são mais intensas que nas fraturas de F3, tornando-as mais susceptíveis a desvios angulares e, até mesmo, rotacionais, circunstância essa que gera uma importante deterioração da funcionalidade dos dedos ao induzir rigidez e redução da amplitude de movimento. Quanto ao tipo de implante de escolha, cada subtipo de fratura de falange média possui um implante de escolha para a fixação, como a combinação de placa e parafuso para as fraturas de diáfise ou parafusos de tração para fraturas condilares, entretanto, no serviço base do estudo, o método de osteossíntese mais acessível era o fio de Kirschner, sendo utilizado na imensa maioria dos casos.

Apresentando a maior incidência dentre as lesões falangianas, as fraturas de F1 não podem ser deixadas de lado, principalmente por sua importância nas articulações interfalangianas proximais (IFP) e metacarpofalangianas (MTCF), responsáveis pelos movimentos de preensão palmar (KAMNERDNAKTA S, et al., 2018). Com distribuição de sexo, idade e mecanismos traumáticos semelhante às fraturas de F3 e F2, as fraturas de $\mathrm{F} 1$ se diferenciam em dois pontos. O primeiro deles é a maior incidência da etiologia traumática ocupacional sobre as causas automobilísticas. Ademais, é perceptível que essas fraturas apresentam uma dificuldade maior em se manterem estáveis devido as forças deformantes que atuam nesse osso, em comparação a F3 e F2.

No que tange ao tratamento das fraturas de F1, suas indicações são semelhantes aos de F2, entretanto, por serem ainda mais susceptíveis às forças deformantes, os métodos de estabilização absoluta como placa e parafusos ganham ainda mais atenção. Isso porque, a estabilidade absoluta diminui a incidência da formação de calos ósseos exuberantes, responsáveis, muitas vezes por diminuir a capacidade de deslizamento dos tendões flexores e extensores. Entretanto, segundo a literatura, nas fraturas extrarticulares, não houve superioridade dos métodos de estabilidade absoluta sobre os fios de Kirschner no que se refere a resultado funcional (EGLOFF C, et al., 2012).

$\mathrm{Na}$ amostra pesquisada, pelos mesmos motivos apontados nas fraturas de F2, o implante de escolha na imensa maioria dos casos foram os fios de Kirschner, apresentando também baixa incidência de complicações. Aqui, como o método adotado é de estabilidade relativa, a imobilização com tala gessada em postura "intrínseco-positiva" associada aos fios de Kirschner foi importante para reduzir a taxa de falhas da osteossíntese ao aumentar o potencial de estabilização do implante (LÖGTERS TT, et al., 2018).

\section{CONCLUSÃO}

Os pacientes que foram analisados nesse estudo apresentaram um perfil com várias semelhanças quando comparados com a literatura existente. Observou-se uma maior prevalência de fraturas no sexo masculino e na idade entre 18 e 60 anos, todos os pacientes foram submetidos à osteossíntese e a grande maioria teve como implante utilizado os fios de Kirschner; em acordo com a literatura. A escolha por esse procedimento cirúrgico, associada ao implante escolhido, relaciona-se com os baixos índices de complicações póscirúrgicas e com menor tempo de internação. Percebe-se que a implementação de protocolos hospitalares baseados em evidências científicas é de suma importância para proporcionar aos pacientes um desfecho mais favorável, e espera-se que esses protocolos sejam amplamente instalados nas unidades hospitalares. 


\section{REFERÊNCIAS}

1. ASPRAY TJ, HILL TR. Osteoporosis and the Ageing Skeleton. Subcell Biochem, 2019; 91(1): 453-76.

2. BLAZAR PE, STEINBERG DR. Fractures of the proximal interphalangeal joint. J Am Acad Orthop Surg, 2016; 8(6): 383-90.

3. EGLOFF C, et al. Ergebnisse osteosynthetisch versorgter Grundphalanxfrakturen der Finger [Results after osteosynthesis of extraarticular proximal phalangeal fractures]. Handchir Mikrochir Plast Chir. 2012; 44(1): 5-10.

4. GERHARDT S, SILVA JB. Trauma do complexo ungueal. Rev Bras Ortop. 2014; 49(2): 111-5.

5. HAWKEN JB, GILADI AM. Primary Management of Nail Bed and Fingertip Injuries in the Emergency Department. Hand Clin., 2021; 37(1): 1-10.

6. KAMATH JB, et al. Conceitos atuais no tratamento de fraturas do metacarpo e falange. Indian J Plast Surg., 2011; 44(2): 203-211.

7. KAMNERDNAKTA S, et al. Complicações das lesões da articulação interfalangeana proximal: prevenção e tratamento. Hand Clin., 2018; 34(2): 267-288.

8. KARL JW, et al. The Epidemiology of Upper Extremity Fractures in the United States, 2009. Journal of Orthopaedic Trauma. 2015; 29(8): e242-e244.

9. KEE C, MASSEY P. Phalanx Fracture. 1르 ed. Treasure Island: StatPearls, 2021.

10. KÖCHLI S, et al. Coordination of bilateral synchronous and asynchronous hand movements. Neurosci Lett, 2020; 720(2): 1347-57.

11. KREMER L, et al. Epidemiology and treatment of phalangeal fractures: conservative treatment is the predominant therapeutic concept. Eur J Trauma Emerg Surg., 2020.

12. LIU S, et al. Percutaneous pinning with interphalangeal joint locked in phalanx fractures: The surgical technique and the results. Medicine (Baltimore), 2021; 100(31): 267-82.

13. LÖGTERS TT, et al. Proximal Phalanx Fracture Management. Hand (N Y)., 2018; 13(4): 376-383.

14. MEALS C, MEALS R. Hand fractures: a review of current treatment strategies. J Hand Surg Am., 2013; 38: 1021-31.

15. PATEL L. Management of simple nail bed lacerations and subungual hematomas in the emergency department. Pediatr Emerg Care., 2014; 30(10): 742-5.

16. RIBAK S, et al. Epidemiology of traumatic injuries of the upper limbs in a university hospital. Acta Ortop Bras., 2018; 26(6): 370-3.

17. ROCKWOOD CA, et al. Fraturas em adultos. $9^{2}$ ed. São Paulo: Manole, 2019.

18. SILVA JB, GERHARDT S. Trauma to the nail complex. Revista Brasileira de Ortopedia (English Edition), 2014; 49(2): $111-115$.

19. SZEKERES M, et al. The Effect of Therapeutic Whirlpool and Hot Packs on Hand Volume During Rehabilitation After Distal Radius Fracture: A Blinded Randomized Controlled Trial. Hand (NY), 2017; 12(3): 265-71.

20. TAHA R, et al. Protocol for surgical and non-surgical treatment for metacarpal shaft fractures in adults: an observational feasibility study. BMJ Open, 2021; 11(6): 469-73

21. WOLFE SW, et al. Green's Operative Hand Surgery [Internet]. Elsevier Health Sciences, 2016. 